\title{
Virtual Reality-based holistic framework: a tool for participatory development of customised playful therapy sessions for motor rehabilitation
}

\author{
Ioannis Th. Paraskevopoulos \\ Department of Computing and Information Systems \\ University of Greenwich \\ London, United Kingdom \\ i.parask@gre.ac.uk \\ Alyson Warland \\ Department of Clinical Sciences \\ Brunel University London \\ London, United Kingdom \\ alyson.warland@brunel.ac.uk
}

\author{
Emmanuel Tsekleves \\ ImaginationLancaster \\ Lancaster University \\ Lancaster, United Kingdom \\ e.tsekleves@lancaster.ac.uk
}

\author{
Cherry Kilbride \\ Department of Clinical Sciences \\ Brunel University London \\ London, United Kingdom \\ cherry.kilbride@brunel.ac.uk
}

\begin{abstract}
The research in-progress presented in this paper proposes a holistic VR-based framework for participatory design of Serious Games for motor rehabilitation. The proposed framework is devised in layers that facilitate the mapping of game mechanics to playful therapeutic exercise games. The framework proposed can be disease agnostic and applies to a wider range of service users, regardless of their condition and motor functional capacity. The pilot study design for evaluating the proposed solution is also presented.
\end{abstract}

Keywords-Virtual Reality, Serious Games, Motor Rehabilitation, home-based, neurological conditions

\section{INTRODUCTION}

Since the introduction of the term Serious Games (SGs) by Ben Sawyer [1] to describe digital games with purpose other than pure entertainment, the field of SGs has been of great research interest. Research and development in this field proliferated and enabled SGs as valid educational and training tools with an exponential curve of number of SGs released every year [2], [3]. Among other application domains, SGs have also been widely used as tools to promote health and wellbeing and condition-specific interventions to target physiotherapy and motor rehabilitation and for a wide span of neurological and degenerative diseases, such as stroke, Parkinson's disease (PD), Multiple Sclerosis (MS) and several others [4]-[7]. The issues created by an ageing population [8], highlight the need for implementation of evidence-based, innovative approaches to empower the service users (SUs) and families to support a continuum of health provision and care; and at the same time, relieve the ever-increasing pressure on respective governmental bodies to provide these services in more cost-efficient ways [9], [10]. Such a sustainable health system, would only be realised by the empowerment of the clinical professional and SU to utilise recently research-evidenced interventions, such as SGs for rehabilitation [11], [12], to create a home-based quiver of solutions and interventions that can be fully customised to fit the rehabilitation targets at request. Customisation needs to take place in all aspects of the design of such interventions, including the therapy regime that needs to be aligned with the game mechanics to maximise the effectiveness of the intervention [13]-[15]. Although there has been evidence as per the engagement, motivational impact [16]-[19] and evidence on effectiveness [4], [6], [7] of SGs for rehabilitation, the proliferation of such interventions and route to market has not been very successful. This is evident by recent research agendas and calls for action issued by international research frameworks, such as the European Research Council [10]. Actions for pre-commercial procurement dictate the need for innovative interventions to reach the commercial level and enable solutions to become widespread, mainstream and available to relevant stakeholders [20]. It is evident that there is a need for holistic approaches at commercial level, encapsulating state-of-the-art technologies for provision of bespoke solutions and interventions. In addition to this and within the context of self-care management, there is a growing need for empowering SUs to become co-creators of healthcare interventions [21]. A significant issue that arises with such an approach is the rate of mismatch of technology advancement with the design, development, research and evidence of such customised interventions. Technology advances rapidly and in the case of SGs for motor rehabilitation, this is reflected in gaming input technologies, game engines, communication technologies and any other technologies used in the process of developing such interventions. As such, there is a need for developing system architecture that would allow a certain extent of flexibility in support and adoptability with, for example, the input technologies for motion capture, or visualisation devices such as Virtual Reality (VR) and 
Augmented Reality (AR) headsets suggesting development of new SGs that would integrate them in their development pipeline. Another significant issue for the further development of next generation of SGs for motor rehabilitation, with an aim for maximising the market penetration, is the participatory design of mini games that underpin motion mechanics directly aligned to the rehabilitation exercises [13], [14]. Finally, through co-creation it is also important to empower relevant stakeholders (clinicians, SUs, families and carers) to develop mini SGs for motor rehabilitation based on individual preferences in terms of content and narrative. Previous research from the authors [13] has shown that game content and narrative is an equally paramount important aspect of designing SGs for rehabilitation to enhance the engagement of the SU with the intervention.

This paper aims to introduce a novel VR prescription of customised therapeutic exercises for home-based SGs interventions for motor rehabilitation and also bind the aforementioned requirements under a proposed framework that would entail the design and development of SGs for motor rehabilitation in a condition and disease agnostic manner. The paper introduces the problem and motivation behind this work and an overview of background studies relevant to the subject matter. This is followed by a presentation of how conventional rehabilitation can be mapped to corresponding game mechanics using a proof of concept; that is then translated into a co-created customised mini game with provision for fully customised and monitored exercise prescription through a VR platform. This is then extrapolated to a conceptual design of the proposed framework, underpinning the requirements mentioned above. Finally, we present the study design for the evaluation of the proposed approach in terms of user engagement and effectiveness.

\section{BACKGROUND WORK}

Efforts on designing and developing games for motor rehabilitation have been the focus of various research centres and individuals over the past years. There is vast corpus of literature relevant to the subject matter of SGs for motor rehabilitation, targeting mostly prominent conditions and diseases such as stroke and PD. The system architecture presented in this paper aims to enable provision of physiotherapy in a disease and condition agnostic manner, offering a holistic approach and solution that can be employed in other motor specific diseases that would require long or short term rehabilitation through exercising with SGs. As such, relevant work presented in this paper focuses and includes work from this scope. The proposed system architecture can be deployed for a wider span of neurological conditions. The conditions mentioned above are used as case studies and are also applicable to previous work conducted by the authors and by the majority of other studies to date.

Stroke is the third most common cause of death after cancer and heart attack, with approximately 152,000 incidents per year in the UK alone [22]. That statistic accounts for about a new incident every 3 minutes and 27 seconds and there are about 1.2 million survivors in the UK according to 2016 statistics. Of those survivors, over a third have strong dependencies to families or carers for their everyday living, whereas half of all stroke survivors face a disability, with deficiencies in the upper limb being the most common.

$\mathrm{PD}$ is a very common progressive neurological disease which primarily affects movement. With one person diagnosed every hour at the UK, it is estimated that a total of 127,000 people are living with PD in the UK [23]. While predominant after the age of 50, PD can present in younger age groups and to date, there is no cure for this disease with treatment being focused on medication and therapies. Physiotherapy is aimed at symptomatic relief and helping retain a healthy and active lifestyle to maximise quality of life. The majority of other neurological conditions require neurorehabilitation and the burden introduced on families, health services and communities by these conditions is projected to be unbearable in the near future [24].

Recent efforts in developing disruptive therapeutic interventions, embodied as SGs for neurorehabilitation, have identified the need for deeper delving into the customisation of the interventions and the need for User Centred Design [25]. The work of Paraskevopoulos and colleagues [11], [13] proposes a system architecture that tackles the game development in layers, as per a more effective and flexible approach to align the system with constant advances of technology and also adhere to provision of customisable and personalised exergames for motor rehabilitation, with performance monitoring and biofeedback at a different layer. The design of the game initiates from the prescribed conventional rehabilitation exercise that is translated into game mechanics and the authors present a series of case studies for Parkinson's rehabilitation. Omelina et al. [26] described a similar system with the game configuration component distinguished from the game itself, thus enabling for flexibility of choice in game controllers and input methods. This enables the customised design of mini games, agnostic of the input technology and the mechanics of motion capture. The authors of the aforementioned study target neurological conditions in general, however, their mini games and game mechanics were restricted to cover selected exercises based on preconfigured parameters for the games. Pirovano et al. [14] present a game design methodology that initiates from exercises which are translated into mini games with primary and secondary rehabilitation aims, in terms of movement within the game. After the primary motion target is designed in VR, the authors of this study tackle the ludic side of the game by adding the aesthetics and other game mechanics, such as the challenge, scores etc. This approach is very similar to that proposed by Paraskevopoulos and Tsekleves [11] in their system architecture. Another study by Epelde et al. [27] proposes a system architecture for the provision of home-based telerehabilitation with customisable features, including multidevice compatibility, tele-monitoring, motion capturing and analysis of high granularity and cloud services. Although this approach doesn't employ SGs per se, as there are no game mechanics implemented, it is rendered as an architecture that follows a coherent approach in terms of the actual requirement 
for a holistic solution for home-based, tele-monitored physiotherapy provision. The distributed architecture presented by [28] proposes a system architecture that is input agnostic as well and also is able to target and deliver motor and cognitive rehabilitation using state of the art human computer interfaces and remote monitoring of the interventions.

Participatory design in healthcare and rehabilitation intervention has been recognized as a promising approach [29], [30] leading to user engagement of the resulting physiotherapy programmes [31]. In particular co-creating with users has the potential to be transformative as participants become co-owners of innovation, having a much higher stake in its design, making and ultimately use [32]. This is true especially when co-creation is coupled with playful design, the mapping of playful experiences from video games to other non-game context experiences [32]. However its implementation within a rehabilitation technology systems approach is often difficult as accessing SUs at the different stages of the design process and appropriate tools to engage them in that is significant. In this paper we posit that the design and development of rehabilitation system architecture and frameworks that encourage co-creation of physiotherapy sections with direct involvement of the therapist and SU is not only possible but also a required function.

In addition, the novelty of the system architecture proposed in this paper lies in its holistic nature, manifest by a number of features. These include the technology agnostic approach, as it tackles the input controllers at a separate layer. It also enables the full customisation of physiotherapy to a higher level of fidelity and granularity as the physiotherapist is able to define the parameters of each repetition of the exercise. This is cocreated with SUs ensuring that the produced rehabilitation programmes embodied within mini games cater for user needs, preferences and wants. Furthermore, there is a clinician platform to monitor and refine the performance. Finally, the game narrative is also fully customizable and can be therefore cocreated with the SU and clinician, as the game input is controlled at a separate layer and the exercise is translated into a game. This enables the interactions and game mechanics to be developed in a ludic agnostic manner, which means that the mini game can be co-created by SUs and clinicians using different content each time.

In the next section, the system architecture is presented with a focus on the VR Physiotherapy Prescription Panel (VRPPP). This is followed by a case study using a pilot game that integrates a conventional rehabilitation exercise translated into a game and the VRPPP in its design and development.

\section{DESIGN OF THE PROPOSED FRAMEWORK}

As discussed in the previous section, the holistic system architecture as a framework to underpin a number of requirements. These are namely:

- Translation of the exercise motion to game mechanics

- Customised motion capture system with established accuracy

- Co-created customised prescription of physiotherapy regime
- Co-created customised game (Content)

- Biofeedback platform (Clinician)

The system design is performed in layers and is depicted in Figure 1 below:

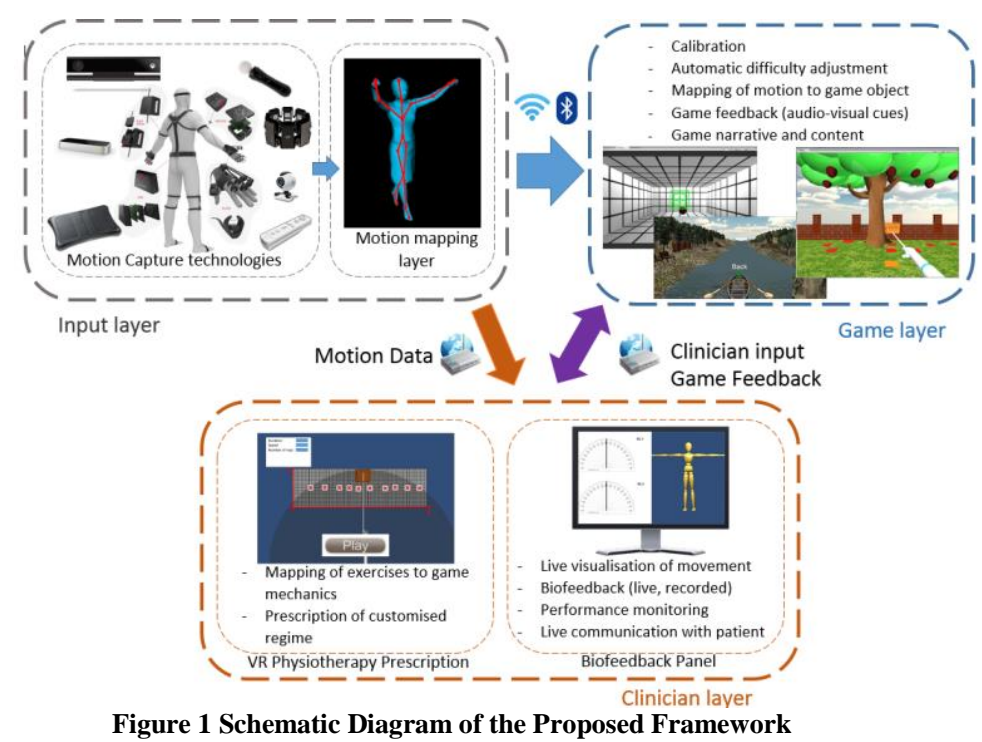

The input layer delivers the capture of motion of the SU to be mapped on an object within the VR environment (mini game). This layer provides a solution that is technology agnostic and enables the intervention to be unaffected by the rapid advances of technology. The motion data are transmitted to the local terminal via Bluetooth or over the Internet. The input technology needs to be of established accuracy as the data captured needs to illustrate and monitor the SU's performance effectively [12]. The motion data are sent over the internet to the clinician platform, as seen in [11]. Once the exercise is translated in to a game, the aesthetics design can take place and the game content is developed in the level accordingly.

Finally, within the clinician layer there is a sublayer, namely the VRPPP. This panel allows the preset of the position of game elements that correspond to interactions in the game but also to the motion extremes of each repetition of the translated exercise. The VRPPP also enables the adjustment of other parameters, such as the whole duration, speed of the game and number of executed repetitions for the exercise. A case study that follows serves as a proof of concept with one mini-game, as translation of an exercise, that can be fully customised from the ludic perspective.

\section{Pilot Game: CASE StUdy}

To assess the feasibility of such an approach, there is a need for developing pilot games as per case studies to investigate the validity of the proposed framework as a viable solution. As described above, the design of the mini game initiates from therapeutic exercises (in this case physiotherapy) that are mapped to game mechanics and then translated into mini games. The VRPPP intermediary panel enables the full customisation of the exercise down to each repetition. 


\section{A. Rehabilitation Protocols to Game Mechanics}

The design of the games needs to initiate from the regime of exercises, conventionally prescribed to the SU for postdischarge rehabilitation. As such, the prescribed set of motor task-specific actions should ideally be translated into game mechanics. That for example, can be the "Reaching Target" exercise seen in a physiotherapy exercise booklet from Physiotherapyexercises.com [33] (Figure 2).

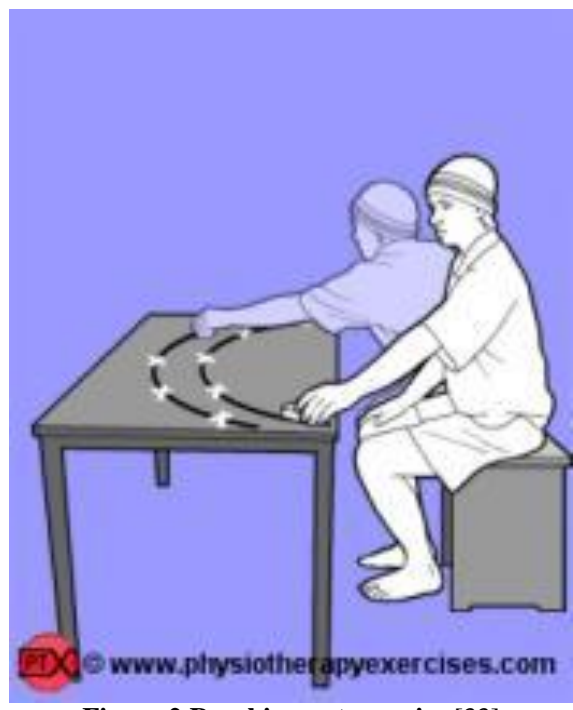

Figure 2 Reaching out exercise [33]

This conventional rehabilitation exercise is set by clinicians to help the SU retrieve and enhance motor capacity in the upper limb by reaching out to predefined targets on a table in front of them. It takes place on the transverse plane of human movement (yaw) and the physiotherapist has to set the targets according to the person's condition and capability.

This type of exercise can be easily co-created into a game with a variety of narratives fitting to cover the ludic aspect of it. In this case the proposed framework functions as a cocreation tool where the SU and therapist can explore different game narratives based on personal needs and preferences. It becomes apparent that the aim of the SG underpinning this motion and exercise is to trigger an event when the person reaches and touches a virtual object in the game environment using the virtual limb that is guided by the game input technology. Following the paradigm of commercial game development pipelines, the design and pre-production phase of this game would output a "grey box prototype" (a prototype of the game with no aesthetics, but only place holders to demonstrate functionality only), where a virtual limb is manipulated in 3D space by the external motion capture hardware (game sensors), and events are triggers (? triggered)when the virtual geometry of the limb collides with another virtual object set in a specific position in the game. In SG design so far, these positions are defined in the design phase and as such there is minimal adjustment that can be made to customise the game. It is in here that lies the value of the proposed framework as a co-creation tool, as enabled by the game design opportunities facilitated within this context. Figure
3 demonstrates the concept of a grey box prototype that would cover the interactions required by this exercise.

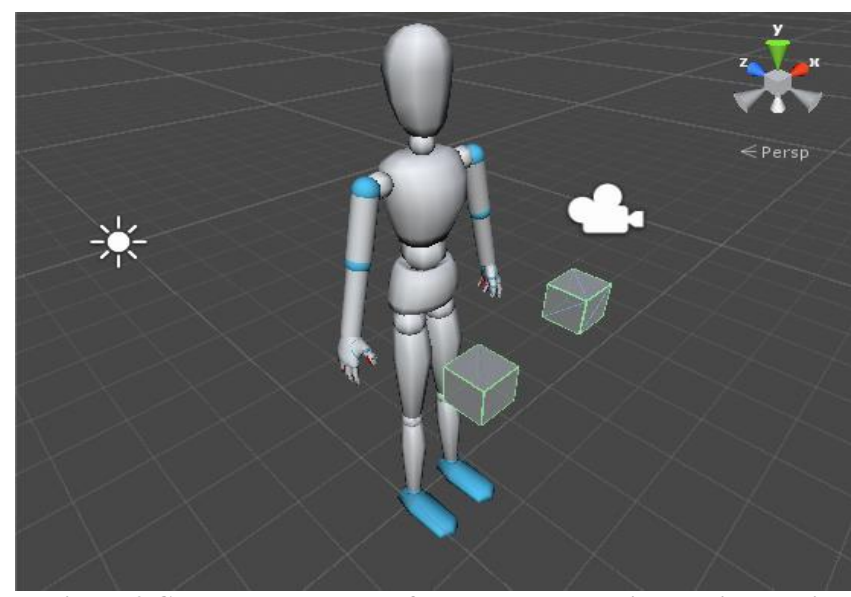

Figure 3 Grey box prototype of the game mechanics and interactions

The virtual avatar in this prototype is fed motion data by the input layer, as seen in Figure 1, and the boxes represent the point that the avatar model interacts with to trigger the game mechanics. These can be a pickup item, an increase in score, a puzzle piece etc. depending on the game narrative and structure. The boxes can also be easily programmed to appear in the game at specific time points or have game physics enabled. The interactions are programmed as triggered by the collision of the avatar with the place holding geometry (gray boxes).

Prior research studies have utilised automatic difficulty adjustment [34], [35], which manipulates the pre-defined positions of the objects in the $3 \mathrm{D}$ game environment to match the condition and playing ability of the player. However, this approach has limitations and although adaptable, cannot be perceived as fully customisable physiotherapy prescription, as it takes place in vivo, within the gameplay. Furthermore, this takes away from SUs critical design decisions that may affect the ultimate acceptance of use of the produced rehabilitation programmes. What is needed is a fully customisable solution to enable the co-creation of the exercises, whereby the physiotherapist can prescribe, a priori, the parameters of each repetition of each exercise based on SU input and individual SU ability. The approach proposed in this paper is the first that enables the co-creation of bespoke physiotherapy regimes for SUs. The challenge is how to classify all available exercises into a taxonomy that can then be mapped to potential game mechanics and be translated into cohorts of mini games that are content and narrative agnostic. Content and narrative can potentially be targeted at a different layer in the development of the game. It can be perceived as developing the game mechanics without any content and afterwards, dealing with the aesthetic aspect of the game by replacing place holding geometry by aesthically pleasing, narrative-specific content.

\section{B. VR Physiotherapy Prescription Panel}

The layered framework as presented in the previous section, requires a tool that would enable the physiotherapist or clinician to prescribe the physiotherapy regime for the specific SU before they play the game. In the paradigm using the "Reaching Target" 
exercise of Figure 1, the clinician would have to define the point that the VR objects would occur in the game environment according to each SU's ability. These would then appear in the game, based on the game mechanics at a specific time and with other properties also able to be adjusted (duration, appearance, speed, physical properties etc.). Figure 4 illustrates the panel that is directly in tune with the "Reaching Target" exercise of Figure 2 .

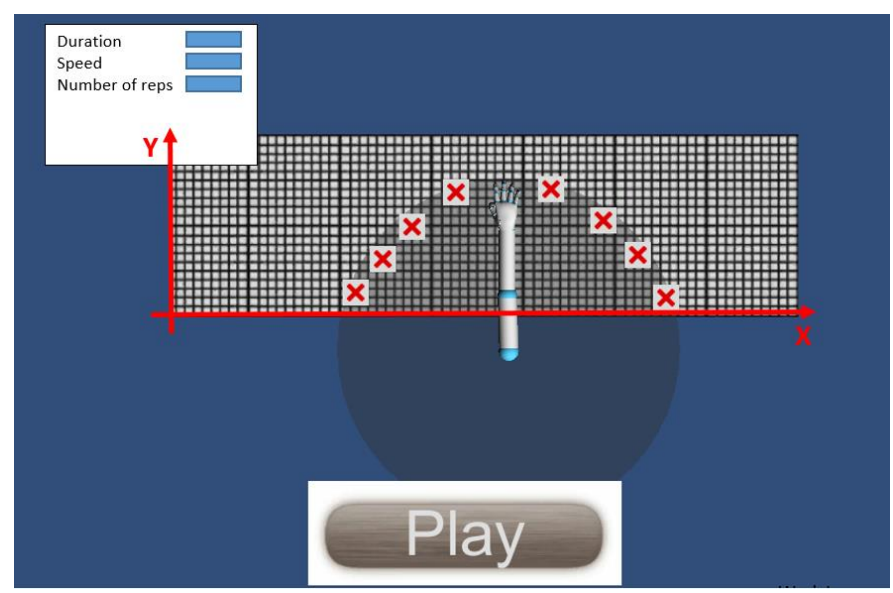

Figure 4 VR Physiotherapy Prescription

The same panel can fit or can be modified to fit other exercise types depending on the plane of motion. Thus, there is a need for a taxonomy of physiotherapy exercises according to the task-specific motion requirements. "Reaching" can be one of the exercise targets and the "Reaching Target" game would be part of this exercise prescription. Other parameters that can be adjusted by this panel include duration of task, speed of execution and number of repetitions. The outcome of this panel is data that are fed to the mini game scene, where the game objects are positioned according to the positioning set by the clinician in the VRPPP. The narrative and concept of the game, thus, is disconnected from the motion capture and interactions in the game. The next task is to develop the narrative concept and ludic aspects of the game. The VRPPP developed for this case study and as depicted above covers all the family of "Reaching Target" exercises and, thus, a number of potential corresponding mini-games.

\section{The Apple Tree Game}

Once the game mechanics and the interactions are in place, as defined through the VRPPP, thereby translating the exercise to a mini game, the next step is to devise meaningful, entertaining and fun narrative concepts to veneer the game mechanics with engaging content. The exemplar exercise employed in this case study is the "Reaching Out" exercise that needs to be translated into a mini game. This is rendered as the main challenge SGs' designers have to face, specifically:

How to translate conventional physiotherapy exercises into mini games for maximising the engagement without affecting the effectiveness of the therapy regime?
The exercise chosen in this case study (and indeed in all similar "reaching-for-an-object" exercises), can be easily translated into some meaningful and engaging intervention in the form of a game. The game shown in Figure 5 below, demonstrates the Apple Tree game developed as a translation of the "Reaching Out" exercise of Figure 2.

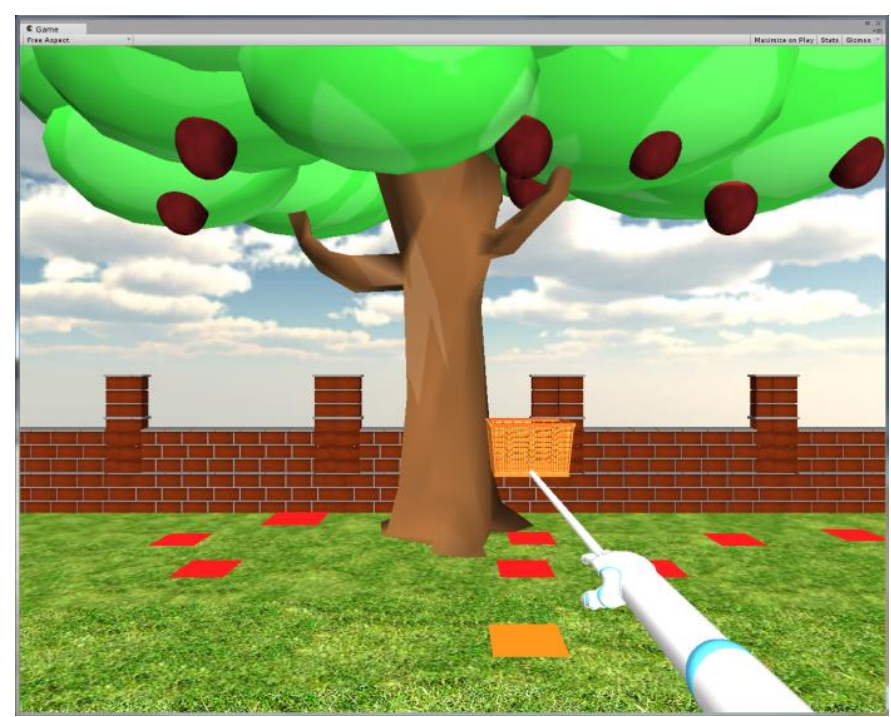

Figure 5 The Apple Tree game prototype

The positions of the apples on the tree are fed into the game through the VRPPP. The positions are mapped onto the virtual apples, with additional physical properties such as speed, number of reps etc. also being adjusted through the VRPPP as are attributes. The game helps the SU to orientate to the VR environment through the use of visual cues that have been found to be a necessary element of design for certain diseases, such as Parkinson's [13].

Thus, the design of the framework has enabled the mapping of technology agnostic motion capture onto a virtual avatar (arm), there is direct mapping and alignment of the exercise targets and game mechanics, the intervention is completely bespoke to the highest level of granularity, down to individual repetitions, and there is provision for optimised engagement through co-created content.

\section{Pilot Study Design}

The aim of this in-progress pilot study is twofold with an ultimate aim of providing a proof-of-concept of the proposed framework. The first aim towards the proof-of-concept is to investigate the user experience and acceptability of the game; and the second is to investigate the clinical effectiveness of the intervention. These aspects of the framework will validate the proof-of-concept and further, more in depth investigation can occur with more games and a wider range of conditions and exercises can be reviewed in the future. The Apple Tree game as a fully customisable therapy session is employed and compared against a fully customisable VR therapy tool that does not have any game mechanics (for more information see [36]). As suggested by [37] in their Cochrane review of studies on VR for stroke rehabilitation, more research is required on 
what type of VR interventions are most effective and how acceptable each approach is to SUs.

Following ethical approval by Brunel University London Ethics Committee, mixed methods (employing an experimental design with a nested qualitative evaluation) is being used to explore issues of acceptability, feasibility and efficacy of use of the device.

Twelve community dwelling unilateral stroke survivors, aged 18 and over, who have completed formal rehabilitation, with mild to moderately severe upper limb weakness (confirmed by Motricity score between 14-25 for shoulder and elbow) have been recruited to the study ( 9 female, 3 male; aged 48-67 years - mean 58 years), 12-304 months post stroke (mean 63.4 months). Four are classified as having moderately severe disability, four as having moderate disability and four as having slight disability (scores of 4,3 and 2 respectively on the Modified Rankin Scale).

Following baseline assessment of impairment (Fugl-Meyer Assessment of motor recovery after stroke), activity (ABILHAND and Action Research Arm Test) and participation (Motor Activity Log-28 and Subjective Index of Physical and Social Outcome), participants will be asked to complete 9, 40 minute exercise sessions using the device over 3 weeks. Participants will be reassessed within one week following the intervention and at a four week follow up. Information regarding enjoyment of activities, level of exertion and any side effects will be collected during the intervention and in addition, participants will complete a semi- structured interview about their study experience. Data will be analysed using non parametric statistical tests (Wilcoxon Signed Ranks and Spearman's Ranks with statistical significance at $p \leq 0.05$ ) and thematic analysis of interview and intervention field-notes.

\section{CONCLUSION AND FUTURE WORK}

This paper presented an in-progress study that aims to investigate the feasibility of a conceptual VR-based holistic framework for participatory design of customised playful therapy interventions for motor rehabilitation. The conceptual framework presented underpins the need for co-created, playful interventions as SGs for maximising the adherence to and engagement with the therapeutic regimes. The framework consists of a fully customisable VR therapy prescription panel, enabling the physician to adjust the regime to a great detail. It also has the ability to send biofeedback data in real time to remote locations. A pilot study is designed and a mini game is developed within the framework proposed to research the acceptability, feasibility and efficacy of the proposed approach. The playful intervention will be compared to a non-ludic VR intervention that is also fully customisable.

To fully assess the validity of the proposed framework, a broader spectrum of exercises and mini-games should be evaluated. Future plans include the further development of tools for the participatory design of the mini-games by stakeholders and the evaluation of the interfaces as well as the effectiveness of the interventions.

\section{ACKNOWLEDGMENT}

The authors of this paper would like to thank the participants of this study for their help and support, and also Hannah Levings and Josephine Gritsci for their assistance in data collection and conducting the pilot study.

\section{REFERENCES}

[1] B. Sawyer, "Serious Games: Improving Public Policy through Game-based Learning and Simulation," Woodrow Wilson International Center for Scholars, 2002.

[2] D. Djaouti and J. Alvarez, "Origins of serious games," Serious games Edutainment Appl., pp. 1-22, 2011.

[3] E. A. Boyle, T. Hainey, T. M. Connolly, G. Gray, J. Earp, M. Ott, T. Lim, M. Ninaus, J. Pereira, and C. Riberio, "An update to the systematic literature review of empirical evidence of the impacts and outcomes of computer games and serious games," Comput. Educ., vol. 94, pp. 178-192, Nov. 2015.

[4] K. R. Lohse, C. G. E. Hilderman, K. L. Cheung, S. Tatla, and H. F. M. Van der Loos, "Virtual reality therapy for adults post-stroke: a systematic review and meta-analysis exploring virtual environments and commercial games in therapy.," PLoS One, vol. 9, no. 3, p. e93318, Jan. 2014.

[5] G. Abbruzzese, R. Marchese, L. Avanzino, and E. Pelosin, "Rehabilitation for Parkinson's disease: Current outlook and future challenges," Park. Relat. Disord., vol. 22, pp. S60-S64, 2016.

[6] N. Skjæret, A. Nawaz, T. Morat, D. Schoene, J. L. Helbostad, and B. Vereijken, "Exercise and rehabilitation delivered through exergames in older adults: An integrative review of technologies, safety and efficacy," Int. J. Med. Inform., vol. 85, no. 1, pp. 1-16, Oct. 2015.

[7] G. Barry, B. Galna, and L. Rochester, "The role of exergaming in Parkinson's disease rehabilitation: a systematic review of the evidence.," J. Neuroeng. Rehabil., vol. 11, no. 1, p. 33, Jan. 2014.

[8] United Nations', "World Population Ageing 2015," Department of Economic and Social Affairs, Population Division: World Population Ageing 2015. 2015.

[9] Department of Health, "Equity and excellence: liberating the NHS (White Paper)," Station. Off., p. 61, 2010.

[10] European Commision, "eHealth Action Plan 20122020 -innvative healthcare for the 21st century," 2011.

[11] I. Paraskevopoulos and E. Tsekleves, "Use of Gaming Sensors and Customised ExerGames for Parkinson 's Disease Rehabilitation," in Games and Virtual Worlds for Serious Applications (VS-GAMES), 2013 5th International Conference on, 2013, pp. 1-5.

[12] E. Tsekleves, A. Warland, C. Kilbride, I. Paraskevopoulos, and D. Skordoulis, "The use of the Nintendo Wii in motor rehabilitation for virtual reality 
interventions: a literature review," in Virtual, Augmented Reality and Serious Games for Healthcare 1, vol. 68, Prof. Minhua Ma, Ed. Springer-Verlag, 2014, pp. 321-344.

[13] I. T. Paraskevopoulos, E. Tsekleves, C. Craig, C. Whyatt, and J. Cosmas, "Design guidelines for developing customised serious games for Parkinson's Disease rehabilitation using bespoke game sensors," Entertain. Comput., Oct. 2014.

[14] M. Pirovano, E. Surer, R. Mainetti, P. L. Lanzi, and N. A. Borghese, "Exergaming and rehabilitation: A methodology for the design of effective and safe therapeutic exergames," Entertain. Comput., p. -, 2015.

[15] G. Abbruzzese, R. Marchese, L. Avanzino, and E. Pelosin, "Rehabilitation for Parkinson's disease: Current outlook and future challenges.," Parkinsonism Relat. Disord., vol. 22, pp. S60-S64, Sep. 2015.

[16] J. W. Burke, M. D. J. McNeill, D. K. Charles, P. J. Morrow, J. H. Crosbie, and S. M. McDonough, "Optimising engagement for stroke rehabilitation using serious games," Vis. Comput., vol. 25, no. 12, pp. 1085-1099, 2009.

[17] P. Wouters, C. van Nimwegen, H. van Oostendorp, and E. D. van der Spek, "A meta-analysis of the cognitive and motivational effects of serious games.," J. Educ. Psychol., vol. 105, no. 2, pp. 249-265, 2013.

[18] T. Susi, M. Johannesson, and P. Backlund, "Serious games: An overview (technical report)," 2007.

[19] P. Rego, P. Moreira, and L. Reis, "Serious games for rehabilitation: A survey and a classification towards a taxonomy," in 2010 5th Iberian Conference on Information Systems and Technologies (CISTI), 2010.

[20] European Commision, "Implementation research for scaling-up of evidence based innovations and good practice in Europe and low- and middle-income countries," Horizon 2020 H2020 website Pillar: Societal Challenges Work Programme Year: H20202016-2017 Work Programme Part: Health, demographic change and well-being Call: H2020SC1-2016-2017, 2015. [Online]. Available: http://ec.europa.eu/research/participants/portal/desktop /en/opportunities/h2020/topics/3056-sc1-pm-212016.html. [Accessed: 19-Mar-2016].

[21] K. Freire and D. Sangiorgi, "Service design \& healthcare innovation : from consumption to coproduction and co-creation," ServDes. 2010 Second Nord. Conf. Serv. Des. Serv. Innov., no. 1993, pp. 111,2010

[22] Stroke.org.uk, "State of the Nation: Stroke Statistics January 2016." 2016.

[23] Parkinson'sUK, 'Parkinson's prevalence in the United Kingdom," 2009. [Online]. Available: http://www.parkinsons.org.uk/pdf/Parkinsonsprevalen ceUK.pdf.

[24] World Health Organization, "Neurological Disorders: Public Health Challenges," Arch. Neurol., vol. 65, no.
1, p. 154, 2008.

[25] J. Perry, J. Andureu, F. I. Cavallaro, J. Veneman, S. Carmien, and T. Keller, "Effective game use in neurorehabilitation: user-centered perspectives," in Handbook of Research on Improving Learning and Motivation through Educational Games: Multidisciplinary Approaches, 2011, pp. 1-44.

[26] L. Omelina, B. Jansen, B. Bonnechère, S. Van, S. Jan, and J. Cornelis, "Serious games for physical rehabilitation: designing highly configurable and adaptable games," in Virtual Reality \& Associated Technologies Laval $\square 2012$ ICDVRAT, 2012, pp. 1012.

[27] G. Epelde, J. Abascal, J. M. Jimenez, K. Vivanco, and I. Gomez-Fraga, "Smart Medical System for the Universal Remote Delivery of Rehabilitation," InImpact J. Innov. Impact, vol. 6, no. 1, p. 98, Jan. 2013.

[28] A. Vourvopoulos, A. L. Faria, M. S. Cameirao, and S. Bermudez I Badia, "RehabNet: A distributed architecture for motor and cognitive neurorehabilitation," in 2013 IEEE 15th International Conference on e-Health Networking, Applications and Services, Healthcom 2013, 2013, pp. 454-459.

[29] J. Clemensen, S. B. Larsen, M. Kyng, and M. Kirkevold, "Participatory design in health sciences: Using cooperative experimental methods in developing health services and computer technology.," Qual. Health Res., vol. 17, no. 1, pp. 122-130, 2007.

[30] L. De Couvreur and R. Goossens, "Design for (every)one : co-creation as a bridge between universal design and rehabilitation engineering," CoDesign, vol. 7, no. 2, pp. 107-121, 2011.

[31] S. Uzor, L. Baillie, and D. Skelton, "Senior designers: empowering seniors to design enjoyable falls rehabilitation tools," Proc. SIGCHI Conf. Hum. Factors Comput. Syst., pp. 1179-1188, 2012.

[32] E. Tsekleves, A. Gradinar, A. Darby, and M. Smith, "Games for Health 2014: Proceedings of the 4th conference on gaming and playful interaction in healthcare," B. Schouten, S. Fedtke, M. Schijven, M. Vosmeer, and A. Gekker, Eds. Wiesbaden: Springer Fachmedien Wiesbaden, 2014, pp. 140-143.

[33] Physiotherapyexercises.com, "Physiotherapy Exercises," Physiotherapy Exercises for people with injuries and disabilities, 2015. [Online]. Available: https://www.physiotherapyexercises.com/.

[34] M. Ma, M. Mcneill, D. Charles, S. Mcdonough, J. Crosbie, L. Oliver, and C. Mcgoldrick, "Adaptive Virtual Reality Games for Rehabilitation of Motor Disorders," Lect. Notes Comput. Sci., vol. 4555, pp. 681-690, 2007.

[35] N. Hocine, A. Gouaich, and S. A. Cerri, "Dynamic difficulty adaptation in serious games for motor rehabilitation," in Lecture Notes in Computer Science (including subseries Lecture Notes in Artificial Intelligence and Lecture Notes in Bioinformatics), 
2014, vol. 8395 LNCS, pp. 115-128.

[36] E. Tsekleves, I. T. Paraskevopoulos, A. Warland, and

C. Kilbride, "Development and preliminary evaluation

of a novel low cost VR-based upper limb stroke

rehabilitation platform using Wii technology.,"

Disabil. Rehabil. Assist. Technol., vol. 3107, no.

February 2016, pp. 1-10, 2014.

[37] K. Laver, S. George, S. Thomas, J. Deutsch, and M.

Crotty, "Virtual reality for stroke rehabilitation

(Review)," Stroke, no. 9, 2012. 